\title{
Evaluación de diferentes dosis de gonadotrofina coriónica equina en el protocolo de sincronización de celo en ovejas
}

\author{
Arbués, R.'; F. Quintana, C. ${ }^{1}$; Yáñez, E. ${ }^{2}$; Kornuta, M. ${ }^{3}$; Fernández, J. ${ }^{3}$ \\ ${ }^{1}$ Cátedra de Histología y Embriología, Facultad Ciencias Veterinarias, Univ.Nac.Nordeste, Cabral 2139, \\ Corrientes, Argentina. ${ }^{2}$ Cátedra de Nutrición y Alimentación, FCV, UNNE. ${ }^{3}$ Cátedra de Nutrición Animal, \\ FCA, UNNE. E-mail: magali.arbues@gmail.com
}

\begin{abstract}
Resumen
Arbués, R.; F.Quintana, C.; Yáñez, E.; Kornuta, M.; Fernández, J.: Evaluación de diferentes dosis de gonadotrofina coriónica equina en el protocolo de sincronización de celo en ovejas. Rev. Vet. 29: 2, 104-108, 2018. Con el objetivo de evaluar los efectos de diferentes dosis de gonadotrofina coriónica equina (eCG) en el protocolo de sincronización de ovejas Santa Inês en el subtrópico argentino, se sincronizaron 55 animales. Las hembras tratadas recibieron esponja intravaginal con progestágeno. El día 5 se retiró la esponja, se aplicó PGF2 $\alpha$ y se dividió a las ovejas en dos tratamientos: T1 recibió 200 UI y T2 300 UI de eCG. Por la tarde las hembras se colocaron con los carneros, iniciándose la observación del servicio. Las variables registradas fueron intervalo al celo y frecuencia de hembras en celo. A los 7 y 30 días del servicio se realizaron ecografías para diagnóstico de cuerpo lúteo (CL) y preñez. El análisis se realizó por tablas de frecuencia, ANOVA y Chi2 ( $\mathrm{p}<0,05)$. El 76,36\% de las ovejas tratadas presentó celo, $85,2 \%$ en T1 y $67,85 \%$ en $T 2$, sin diferencia significativa ( $p>0,05$ ). En cambio, el intervalo al celo fue diferente entre tratamientos $(\mathrm{p}<0,05)$. En $\mathrm{T} 1$ los celos se concentraron entre las 48 y $60 \mathrm{~h}$ de finalizado el mismo, mientras que en T2 ocurrió entre 24 a $36 \mathrm{~h}$. De aquellas hembras a quienes se pudo localizar el ovario mediante ecografía, en T1 79\% presentaron 1 CL, 10,5\% 2 CL y 10,5\% solamente folículos. En T2 estos porcentajes fueron $69 \%, 25 \%$ y $6,3 \%$ respectivamente ( $>0,05)$. En cuanto a la gestación, en T1 el 66,6\% tuvo preñez simple y el 11,11\% doble, mientras que en T2 los valores fueron $60,71 \%$ y $17,9 \%$ para preñez simple y doble respectivamente ( $>0,05)$. Se concluye que en ovejas Santa Inês de la región subtropical, las dosis de 200 y 300 UI de eCG permiten obtener porcentajes de celo superiores al $67 \%$ y que las dosis de 300 UI adelantan la presentación de celo.
\end{abstract}

Palabras claves: ovino, subtrópico argentino, estro, cuerpo lúteo, ecografía.

\begin{abstract}
Arbués, R.; F.Quintana, C.; Yáñez, E.; Kornuta, M.; Fernández, J.: Evaluation of different doses of equine chorionic gonadotropin in the protocol of heat synchronization in sheep. Rev. Vet. 29: 2, 104-108, 2018. $\mathrm{n}$ order to evaluate different doses of equine chorionic gonadotropin effects in the synchronization protocol of Santa Inês ewes in subtropical Argentina, 55 sheep were synchronized. Treated females received intravaginal sponges containing progestagen for 5 days, after this period sponges were withdrawn and animals were divided into two treatments. T1 received 200 and T2 300 UI de eCG, respectively. In the afternoon sheeps and rams were located together and observation of the service began. The parameters assessed were interval to heat and frequency of females in heat. Seven and thirty days after the service ultrasound was performed to determine the presence of corpus luteum $(\mathrm{CL})$ and gestation. The statistical analysis was made by frequency tables, ANOVA and Chi2 $(\mathrm{p}<0,05)$. Results indicate that $76,36 \%$ of treated ewes showed estrus, $85,2 \%$ in $\mathrm{T} 1$ and $67,85 \%$ in T2, with no significant difference $(\mathrm{p}>0,05)$. The interval to heat was different between treatments $(\mathrm{p}<0,05)$. In the $\mathrm{T} 1$ estrous were concentrated between 48 and $60 \mathrm{~h}$ after its end, while in T2 this occurred between 24 and $36 \mathrm{~h}$. From those females that ovary could be located by ultrasound, in T1 79\% presented $1 \mathrm{CL}, 10.5 \% 2 \mathrm{CL}$ and $10.5 \%$ only follicles; for $\mathrm{T} 2$ these percentage were $69 \%, 25 \%$ and $6,3 \%$, respectively (p>0,05). Regarding pregnancy, in $\mathrm{T} 166.6 \%$ had simple pregnancy and $11.11 \%$ had double pregnancy, while in T2 values were $60.71 \%$ and $17.9 \%$ for single and double pregnancy, respectively $(p>0,05)$. It is concluded that, in Santa Inês ewes of Argentinean subtropical region, doses of 200 and 300 IU of eCG allow to obtain percentages of estrus superior to $67 \%$, and that $300 \mathrm{IU}$ cause the presentation of earlier estrus.
\end{abstract}

Key words: ewe, argentine subtropical, small ruminant, estrous, corpus luteum, ultrasound. 


\section{INTRODUCCIÓN}

La sincronización de celo en pequeños rumiantes tiene como objetivo concentrar las pariciones en épocas deseables o inducir la actividad reproductiva en etapas de anestro ${ }^{9}$. Los métodos más usados involucran a los progestágenos, la prostaglandina y la gonadotrofina coriónica equina (eCG).

Las dos primeras hormonas actúan imitando o destruyendo al cuerpo lúteo, respectivamente. Los progestágenos se utilizan frecuentemente en ovinos, su principal actividad es la de suprimir el celo y la ovulación a través del mecanismo de retroalimentación negativa sobre la liberación de hormona liberadora de gonadotropina $(\mathrm{GnRH})$ y gonadotropinas ${ }^{4}$. Se administran a través de esponjas de poliuretano aplicadas intravaginalmente.

Las esponjas se emplean masivamente en tratamientos largos de 12 a 14 días de duración pero los resultados son dispares. Si bien la tasa de inducción de celo es alta, la fertilidad es muy variable y por ello algunos protocolos proponen mantenerla por menos días ${ }^{12}$.

Aunque existen varios factores externos que pueden afectar la fertilidad de la hembra luego de estos tratamientos, la baja fertilidad podría estar relacionada con los efectos que ejercen los progestágenos sobre la dinámica de crecimiento folicular.

Otra hormona utilizada frecuentemente en programas de sincronización es la eCG. El útero equino secreta esta gonadotrofina placenaria ${ }^{8}$. Al ser empleada en especies diferentes a la yegua, tiene efecto luteinizante ( $\mathrm{LH})$ y folículo estimulante (FSH), aumentando la ocurrencia y velocidad de la ovulación, favoreciendo que éstas se produzcan en un intervalo de tiempo más corto ${ }^{10,13}$.

En general los protocolos consisten en aplicación de esponjas o dispositivos durante cierta cantidad de días. Al momento del retiro de éstos últimos se administra una dosis de prostaglandina (PGF2 $\alpha$ ) y otra de eCG. Las dosis a emplear varían dependiendo de la edad del animal, de la temporada reproductiva y de la raza. La eCG debe administrarse con precaución ya que provoca el aumento de la tasa de ovulación ${ }^{1}$.

Las menores dosis son usadas en razas prolíficas puesto que su aplicación incrementa la ocurrencia de partos gemelares y esto demanda un mayor requerimiento metabólico de la oveja, así como un buen desarrollo de su habilidad materna ${ }^{15}$. Estudios realizados con ovejas Pelibuey en condiciones de trópico demostraron que las dosis de 200 y 250 UI de eCG indujeron altos porcentajes de ovejas en estro, aunque existió diferencia significativa a favor de la dosis mayor ${ }^{2}$.

Por otra parte, algunos estudios indicaron que con el uso repetido de esta hormona puede disminuir la fertilidad debido al desarrollo de anticuerpos. En cabras, la respuesta inmunológica a la eCG es variable entre individuos ${ }^{14}$. En ovejas con reiteradas aplicaciones de eCG, la presencia de inmunoglobulinas es contro- versial. La reducción al mínimo de la dosis efectiva de eCG representa una ventaja también en este punto.

La respuesta a tratamientos de sincronización de celo en "ovejas de pelo" en condiciones de trópico, generalmente es alta ${ }^{13}$. La evaluación de la respuesta a los protocolos de sincronización en hembras de estas razas -en regiones situadas a mayores latitudes- resulta de sumo interés, ya que permite optimizar el uso y la dosificación de la eCG.

El objetivo del trabajo fue evaluar los efectos de diferentes dosis de eCG en el protocolo de sincronización en ovejas Santa Inês, en el subtrópico argentino.

\section{MATERIAL Y MÉTODOS}

El ensayo se realizó durante el otoño en el establecimiento agropecuario "Don Donato" (Monte Caseros, Corrientes). Su localización es $30^{\circ} 30^{\prime} \mathrm{S}$ y $58^{\circ} 02^{\prime} \mathrm{O}$, registrando un clima subtropical húmedo

Se emplearon 55 ovejas Santa Inês con peso promedio de $43 \mathrm{~kg}$ y condición corporal entre 2 y 4 en la escala del 1 al 5. Las hembras eran sexualmente maduras y no gestantes. Para el servicio se utilizaron 5 machos Santa Inês con peso promedio de 71,5 kg. Todos los animales recibieron manejo cuidadoso y estricto control sanitario a fin de garantizar la eficiencia reproductiva tanto en machos como en hembras.

El esquema de sincronización de celo fue el siguiente: el día 0 se colocó a todas las hembras esponja intravaginal con acetato de medroxiprogesterona, 60 $\mathrm{mg}$. El día 5 se retiró la esponja y se aplicaron $75 \mathrm{mcg}$ de PGF $2 \alpha$.

Luego, las ovejas se dividieron en dos tratamientos: el grupo T1 recibió 200 UI de eCG por vía intramuscular y el grupo T2 300 UI de eCG por la misma vía. El día 5 por la tarde se colocaron las ovejas de ambos tratamientos con los carneros y se inició la observación del servicio para detectar hembras en celo.

Para identificar a las ovejas de cada tratamiento se utilizó pintura para detección de celo (Celotest). A los machos se les colocaron pigmentos inorgánicos (ferrite) de color amarillo por delante del prepucio, a fin de que éstos marquen a las hembras al montarlas.

Para la observación del servicio de detección de ovejas en celo, hembras y machos se colocaron en potreros adecuados para la visualización de los animales. La observación se inició a las $24 \mathrm{~h}$ de retirada la esponja y aplicada la eCG y finalizó a las $82 \mathrm{~h}$, comenzando cada día a las 7 de la mañana y hasta las 7 de la tarde.

La hembra se consideró en celo cuando aceptó la cópula del carnero o presentó algún comportamiento que indique celo, como buscar la compañía del carnero o quedarse quieta junto él ${ }^{6}$; también por la presencia de ferrite en la zona de la grupa o la pérdida del color del Celotest. Al momento de observar una o más de estas tres variables, se anotó en planillas la caravana de la oveja, la fecha y la hora.

A partir de los datos obtenidos durante la observación del servicio se determinó: a) intervalo al celo (ho- 
ras transcurridas desde la finalización del tratamiento de sincronización hasta la observación de síntomas de celo) y b) frecuencia de hembras en celo (porcentajes de hembras en celo en cada intervalo de tiempo). Este último dato resultó de dividir el tiempo total de observación en periodos de $12 \mathrm{~h}$, quedando de la siguiente manera: entre 24 y 36 h; entre 36 y 48 h; entre 48 y 60 $\mathrm{h}$; entre 60 y $72 \mathrm{~h}$ y entre 72 y $82 \mathrm{~h}$.

A los 7 y 30 días del servicio se realizaron ecografías para observar cuerpo lúteo y diagnosticar preñez respectivamente. Los resultados se analizaron estadísticamente por medio de tablas de frecuencia, análisis de la varianza y método $\mathrm{Chi}^{2}(\mathrm{p}<0,05)$, utilizando el programa Infostat $2013{ }^{5}$.

\section{RESULTADOS Y DISCUSIÓN}

Hembras en estro. Durante el periodo observado, el $76,36 \%$ de las ovejas tratadas presentó celo, $85,2 \%$ en T1 y $67,85 \%$ en T2. No obstante, el análisis estadístico no arrojó diferencia $(p>0,05)$. En cambio, el intervalo al celo fue diferente entre tratamientos $(p<0,05)$, como se detalla en Tabla 1. Las frecuencias de hembras en celo por periodos de $12 \mathrm{~h}$ se presentan en la Figura 1.

Observación del cuerpo lúteo. En las ovejas T1 (200 UI de eCG) se pudo localizar el ovario mediante ultrasonografía en el 70,4\% (19/27), en tanto en las hembras T2 (300 UI de eCG) este porcentaje fue 57,14\% (16/28). Los resultados se presentan en la Tabla 2.

Del total de hembras observadas en celo en ambos tratamientos, se pudo constatar ovulación mediante la observación de CL en el $62 \%$ de las mismas. Existió un $11 \%$ de ovejas que no fueron observadas en celo y $\sin$ embargo presentaron CL.

Posiblemente, tales hembras tuvieron celo demasiado corto o bien fueron servidas durante la noche o en momentos en los cuales la observación no se estaba realizando, quizás juntamente con algún problema en la identificación con pinturas en estos pocos casos, ya que de estas hembras, más de la mitad resultaron preñadas.

Por otra parte, algunas hembras que si fueron detectadas en celo, en la ecografía solo presentaron folículos. En el análisis por tratamientos, en T1 se detectó celo y CL en el $55,55 \%$ de las ovejas, en tanto que en T2 este porcentaje fue $39,2 \%$ (Figura. 2).

Gestación. Considerando el total de ovejas tratadas, el $78 \%$ de las mismas resultaron preñadas. Los detalles del análisis se presentan en la Tabla 3.

Los porcentajes de preñez en ambos tratamientos no fueron significativamente diferentes. $(p>0,05)$. Al relacionar las variables estudiadas (porcentaje de ovejas en celo, porcentaje de ovejas con o sin CL, porcentaje de ovejas preñadas) se observa que del total de las hembras del ensayo, en el 38,2\% de ellas estas tres variables coincidieron, es decir que fueron vistas en celo, luego se observó CL en la ecografía y finalmente estas ovejas resultaron preñadas. Al discriminar por tratamientos, en T1, este porcentaje fue $44,44 \%(12 / 27) \mathrm{y}$ en T2 fue $32,1 \%(9 / 28)$.
Tabla 1. Porcentaje de ovejas en celo y promedio de intervalo sincronización-celo en horas, en ambos tratamientos.

\begin{tabular}{lccc}
\hline tratamiento & $\mathrm{n}$ & ovejas en celo (\%) & intervalo \\
\hline 200 UI de eCG & 27 & $85,2(23 / 27)$ & $53,46 \mathrm{a}$ \\
300 UI de eCG & 28 & $67,85(19 / 28)$ & $43,84 \mathrm{~b}$ \\
\hline
\end{tabular}

eCG: gonadotrofina coriónica equina. Intervalo: etapa sincronización-celo (horas). Letras diferentes en una misma columna indican $\mathrm{p}<0,05$.

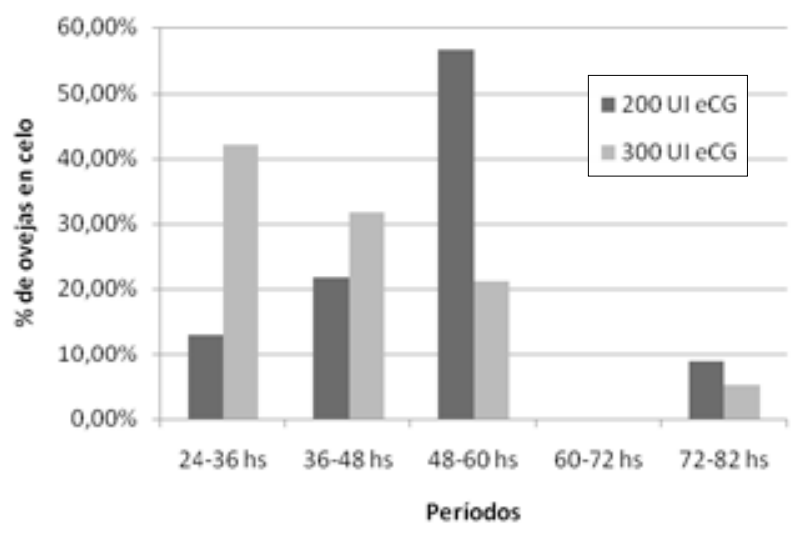

Figura 1. Frecuencias de ovejas en celo agrupadas en intervalos de $12 \mathrm{~h}$ desde el retiro de las esponjas, en ambos tratamientos.

Otros investigadores compararon dosis de 300 y 500 UI de eCG en ovejas lanadas en anestro, sin encontrar diferencias significativas en el porcentaje de celo pero sí en el intervalo al celo, que fue más largo al emplear la menor dosis ${ }^{3}$. El porcentaje de preñez, fue mayor al usar 500 UI. En cuanto a las gestaciones múltiples, se observó ligero aumento al aplicar esta última dosis. Cabe aclarar que el porcentaje de preñez fue del $20 \%$ al usar 300 UI, valor muy bajo en comparación con los resultados de otros autores y los del presente ensayo. En este caso, podría pensarse en que algún otro factor también influyó en el porcentaje de preñez.

En otros trabajos se probaron dosis de 150 y 300 UI de eCG en ovejas cruza Merino por Damara, raza de pelo, en otoño e invierno ${ }^{11}$. No se encontró diferencia significativa en el porcentaje de hembras en celo ni en el intervalo al celo. No obstante, hubo una tendencia a acortarse a medida que la dosis aumentó y ocurrió una mayor agrupación en la presentación de los mismos al usar la mayor dosis. La fertilidad fue de $40 \%$ al usar 150 UI de eCG y de 46,6\% al usar 300 UI, tampoco diferentes estadísticamente. Estos valores son más bajos que los aquí obtenidos (T1: 77,7\% y T2: 78,6\%), pudiendo atribuirse al empleo de dispositivos de progesterona por 12 días, lo que pudo haber reducido la fertilidad. Además, en la otra investigación los autores realizaron IA por laparoscopía y determinaron fertilidad al momento del parto y no por ultrasonografía.

En el nordeste mejicano se compararon protocolos con esponja intravaginal por 10 días y $0,100,200$ y 400 UI de eCG y servicio natural a ovejas Black Belly 
Tabla 2: Frecuencias de ovejas con uno y dos cuerpos lúteos y únicamente con folículos.

\begin{tabular}{lcccc}
\hline tratamiento & $\mathrm{n}$ & $1 \mathrm{CL}$ & $2 \mathrm{CL}$ & solo folículos. \\
\hline $200 \mathrm{UI}$ eCG & 19 & $78,90 \%(15 / 19)$ & $10,52 \%(2 / 19)$ & $10,52 \%(2 / 19)$ \\
$300 \mathrm{UI} \mathrm{eCG}$ & 16 & $68,75 \%(11 / 16)$ & $25 \%(4 / 16)$ & $6,26 \%(1 / 16)$ \\
\hline
\end{tabular}

eCG: gonadotrofina coriónica equina, CL: cuerpo lúteo.

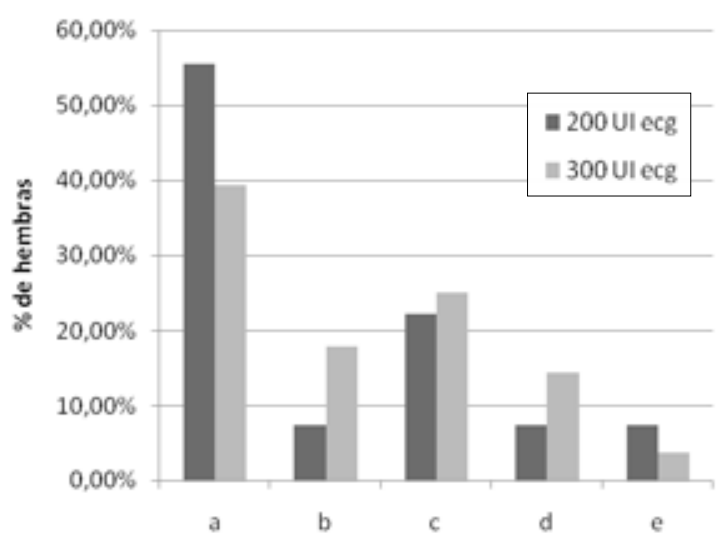

Figura 2. Frecuencia de ovejas en las cuales se detectó celo y cuerpo lúteo (a); no se observó celo ni cuerpo lúteo (b); se observó celo pero no cuerpo lúteo (c); no se observó celo pero sí cuerpo lúteo (d); se observó celo y únicamente ovario (e).

Tabla 3. Porcentajes de ovejas con gestación simple, doble y ovejas vacías con dos dosis diferentes de gonadotrofina coriónica equina (eCG).

\begin{tabular}{lccc}
\hline tratamientos & simples & dobles & vacías \\
\hline $200 \mathrm{eCG}$ & $66,60 \%$ & $11,11 \%$ & $22,22 \%$ \\
$300 \mathrm{eCG}$ & $60,71 \%$ & $17,90 \%$ & $21,42 \%$ \\
\hline
\end{tabular}

y Pelibuey a $24^{\circ} \mathrm{LN}$ y se analizó el porcentaje de ovejas en estro en diferentes periodos luego de la extracción de las esponjas ${ }^{13}$. Dentro de las $24 \mathrm{~h}$ las hembras control y las que recibieron 100 y 200 UI respondieron de manera similar, mientras que las que recibieron $400 \mathrm{UI}$ tuvieron una mayor respuesta. Entre las 24 y $36 \mathrm{~h}$ no hubo efecto de la dosis y finalmente entre las 36 y 48 $\mathrm{h}$ la mayor respuesta se dio en las hembras control. La dosis de eCG no afectó el porcentaje total de hembras en celo, la tasa de preñez ni el porcentaje de gestaciones simples o múltiples, de forma semejante a nuestros resultados.

No obstante, aunque sin diferencias significativas, el mayor porcentaje de gestaciones múltiples, se presentó al aplicar la mayor dosis de eCG, lo cual también ocurrió en el presente estudio. De todas formas, la respuesta "superovulatoria" al tratamiento con gonadotropina es altamente variable, dependiendo además de factores como etapa del ciclo estral, sincronía de la onda folicular, estatus nutricional y estación ${ }^{16}$.

Por su parte, otros evaluaron el efecto de 200 y 250 UI de eCG sobre porcentajes de celo y de gestación en ovejas Pelibuey en una región tropical de otoño a primavera $^{2}$. Encontraron diferencias significativas entre tratamientos en ambas variables estudiadas $(p<0,05)$. Al analizar la razón de la diferencia significativa entre las dosis empleadas, surgió la importancia del tiempo de observación de ovejas en celo. En raza Pelibuey se detectó celo durante las $48 \mathrm{~h}$ posteriores al retiro del progestágeno, en tanto que en el presente trabajo, en hembras Santa Inês, se observó durante las $82 \mathrm{~h}$ posteriores. Con respecto a la diferencia en el porcentaje de gestación encontrada entre tratamientos, ella puede atribuirse al uso de inseminación artificial intrauterina, ya que en un ensayo con servicio natural no se hallaron diferencias entre dosis de 100 , 200 y 300 UI de eCG en el porcentaje de preñez ${ }^{13}$.

En ovejas de pelo se evaluaron dosis de 0, 200, 300 y 400 UI de eCG más esponja con acetato de fluorogestona (FGA) durante 12 días, en primavera ${ }^{7}$. Se encontraron diferencias $(p<0,05)$ en el porcentaje de estro entre 0 y 200 UI de eCG. Entre 200 y 300 UI no hubo diferencia en esta variable, de manera similar a lo ocurrido en el presente ensayo. Sin embargo, el porcentaje de celo se redujo. En cuanto al intervalo al celo, distinto a lo ocurrido en el presente ensayo, no se encontraron diferencias significativas usando 200, 300 o 400 UI pero sí al no aplicar la droga. Al analizar el efecto del genotipo sobre las mismas variables, el Pelibuey Blanco fue quien obtuvo mayor porcentaje de hembras en celo $(p<0,05)$.

En el presente trabajo no se encontró diferencia significativa entre tratamientos en el porcentaje de gestaciones múltiples, aunque las gestaciones dobles no superaron el $20 \%$ y no existieron gestaciones triples. Con el uso de 300, 450 y 600 UI de eCG en ovejas durante el anestro, la prolificidad se incrementó al usar las dos dosis más altas ${ }^{17}$. Esto sugiere que las dosis de 300 UI de eCG deben ser empleadas cuando el objetivo del tratamiento sea sincronizar el momento de aparición del estro y obtener buena fertilidad.

Por lo discutido hasta aquí, se refleja que los estudios que evaluaron dosis diferentes de eCG en protocolos de sincronización presentan resultados variables, observándose, solo en algunos casos, diferencias significativas entre tratamientos. Muchas veces no se evalúan las mismas variables o éstas no son registradas en el mismo momento o durante igual cantidad de tiempo. No es lo mismo emplear servicio natural que inseminación artificial, se debe considerar que en la especie ovina existe el efecto macho, lo que puede influir en el inicio del celo. Además, esta especie presenta una fuente adicional de variabilidad que es la estacionalidad reproductiva.

Se concluye que en ovejas de raza Santa Inês afincadas en regiones subtropicales, es posible diseñar los protocolos de sincronización de celo empleando esponjas con progestágenos durante 5 días, obteniéndose buenos resultados. Esto permite cierta flexibilidad para programar los trabajos en el campo. Dosis de 200 y 300 UI de eCG permiten obtener porcentajes de hembras en celo superiores al $67 \%$, con un nivel manejable de 
gestaciones dobles. Finalmente, según se vaya a realizar servicio natural o inseminación artificial, se debe tener en cuenta que las dosis de $300 \mathrm{UI}$ adelantaron la presentación del celo.

Agradecimientos. A la Sra. Mabel Zambón de Yáñez, propietaria del establecimiento, por su valiosa colaboración. A GesGan Asesores SRL, por aportar los animales e insumos necesarios para el desarrollo del trabajo.

\section{REFERENCIAS}

1. Abecia J, Forcada, F, González BA. 2011. Pharmaceutical control of reproduction in sheep and goats. Vet Clin North Am - Food Anim Pract 27: 67-79.

2. Aké J, Centurión F, Magaña J, Aké V. 2014. Efecto del progestágeno y de la dosis de gonadotropina coriónica equina en la sincronización del estro y tasa de gestación en ovejas Pelibuey inseminadas por laparoscopia. Ecosist \& Rec Agropec 1: 261-268.

3. Catalano R, Teruel M, Cabodevila J, Callejas S. 2007. Efecto de diferentes dosis de gonadotropina coriónica equina sobre la respuesta reproductiva de hembras con un tratamiento para inducción de celos. Investig Vet 9: 11-17.

4. Cordero J et al. 2011. Reducción de dosis de acetato de fluorogestona mediante partición de esponjas para sincronización del estro en ovejas. Rev Cientif FCV-LUZ 21: 492-499.

5. Di Rienzo J, Casanoves F, Balzarini M, González L, Tablada M, Robledo C. 2013. InfoStat versión 2013. Grupo InfoStat, FCA, UNC. URL: http://www.infostat.com.ar

6. Fraser A. 1980. Comportamiento de los animales de granja, $2^{\mathrm{a}}$ ed., Ed. Acribia, Zaragoza, España, 291 p.

7. Gonzalez RA et al. 2014. Factores que alteran la conducta del estro en ovejas de pelo sincronizadas con acetato de fluorogestona y gonadotropina de suero de yegua preñada. AbanicoVet 4: 13-20.
8. Hafez E, Hafez B. 2002. Reproducción e inseminación artificial en animales, McGraw-Hill, México, 33 p.

9. Letelier $\mathbf{C}$ et al. 2009. Ovarian follicular dynamics and plasma steroid concentrations are not significantly different in ewes given intravaginal sponges containing either 20 or $40 \mathrm{mg}$ of fluorogestone acetate. Teriogenology 71 : 676-682.

10. Martemucci G, Alesandro DA. 2010. Estrous and fertility responses of dairy ewes synchronized with combined short-term GnRH, PGF2 $\alpha$ and estradiol benzoate treatments. Small Rum Res 93: 41-47.

11. Martínez TJ et al. 2006. Efecto de eCG e inseminación laparoscópica sobre el comportamiento reproductivo en ovejas F1 (Damara x Merino), Rev Cient (Maracaibo) 16: 72-77.

12. Prieto M, García MG, Lateulade I, Villa M. 2010. Sincronización de celos en ovinos con doble dosis de prostaglandina. www.producción-animal.com.ar

13. Quintero EJ. et al. 2011. The effects time and dose of pregnant mare serum gonadotropin (PMSG) on reproductive efficiency in hair sheep ewes. Trop Anim Health \& Prod 43: 1567-1573.

14. Roy $\mathbf{F}$ et al. 1999. Humoral immune response to equine chorionic gonadotrophin in ewes: Association with major histocompativility complex and interference with subsequent fertility. Biol Reprod 61: 209-218.

15. Silva M. 2012. Uso de la combinación de melatonina y prostaglandinas para la sincronización del estro en ovejas de raza "rasa aragonesa". Tesis de Maestría, Facultad de Ciencias Veterinarias, Universidad de Zaragoza, España, $69 \mathrm{p}$.

16. Wildeus S. 2000. Current concepts in synchronization of estrus: sheep and goats. J Anim Sci 77, 1-14.

17. Zaiem I, Tainturier D, Chemli J, Soltani M. 1996. Vaginal sponges and different PMSG doses to improve breeding performances of Black Thibarewes. Rev Med Vet 147: 305-310. 